\title{
SN démonstratifs et anadeixis: sens 'spatial', ou valeurs tributaires d'une stratégie pragmatique potentielle? 1
}

\author{
FRANCIS CORNISH
}

Université de Toulouse Jean Jaurès

(Received April 2015; revised September 2015)

\section{RÉS U MÉ}

L'article vise à démontrer que l'emploi des démonstratifs marqués pour la distinction déictique 'proximal' vs. 'distal' en français et en anglais (ainsi que dans bien d'autres langues encore) ne se laisse pas analyser de manière satisfaisante en fonction du degré de proximité ou de distance du référent visé par rapport au locuteur (ou à l'allocutaire) dans la situation de communication, selon la conception classique. Une analyse plus satisfaisante devrait prendre en compte le projet communicatif du locuteur: implication personnelle forte du locuteur dans le cas du membre proximal de l'opposition, et dissociation subjective ou alignement avec l'allocutaire dans celui du membre distal (non marqué).

\section{IN TRODUCTION}

Cet article porte sur le fonctionnement énonciatif et discursif des marqueurs démonstratifs nominaux à portée proximale vs. distale en français et en anglais, ainsi que sur la manière la plus appropriée de le décrire. Nous commencerons par un bref rappel des propriétés des expressions démonstratives marquées pour la distinction déictiquement pertinente 'proximal' vs. 'distal'. Par là, nous entendons le marquage morphologiquement distinctif (voir ci-dessous) des formes démonstratives concernées: le présent article a précisément pour but de démontrer que les valeurs portées par des expressions munies de l'une ou l'autre de ces marques ne sont pas d'ordre essentiellement spatial, mais plutôt d'ordre " perspectival ", mettant en jeu le contrôle par le locuteur de ses rapports avec l'interlocuteur ainsi qu'avec l'objet de discours désigné.

Ce $N$-ci, celui-ci en français, et this $(N)$ en anglais sont les membres marqués des paires concernées, les membres distaux (ce $N$-là, celui-là et that $(N)$ ) étant non marqués. Lors de leur emploi, les démonstratifs nominaux manifestant la marque distinctive 'proximale' présentent l'information véhiculée au sein des SN en

${ }^{1}$ Je tiens à remercier pour leurs remarques sur les versions préliminaires de cet article, ou bien sur celle soumise à la revue, les trois relecteurs anonymes, ainsi que Céline Guillot, Walter De Mulder, Fabio Del Prete, et surtout Marie-José Béguelin. 


\section{Francis Cornish}

question comme non présupposée. Plutôt, leur nom tête (accompagné le cas échéant de modificateurs, de compléments etc.) sert à classifier le référent visé en fonction de la perspective subjective du locuteur; cette classification ne se présente pas comme 'allant de soi' vis-à-vis de l'objet de la référence lui-même. Cela n'est pas habituellement le cas des noms têtes (éventuellement avec expansion) au sein des SN définis ou possessifs: la catégorie d'entités qu'ils dénotent est normalement présupposée s'appliquer à leur référent (cf. aussi Guillot, 20I3 pour le français médiéval).

Quant aux démonstratifs nominaux distaux, le statut de leur tête lexicale serait par hypothèse à mi-chemin entre le caractère présupposé des noms têtes des SN définis, et celui non présupposé de leurs homologues dans les SN démonstratifs proximaux: tantôt ces noms têtes le sont, tantôt non (voir la sous-section 2.2 à cet égard).

Par ailleurs, les expressions définies réferent de façon 'inclusive', alors que les démonstratifs le font de manière 'exclusive': leur emploi implique qu'il y a d'autres entités du même type qui ne sont pas comprises dans l'ensemble d'entités qu'ils dénotent. Voir Diessel (I999) pour une vue d'ensemble approfondie des démonstratifs dans les langues du monde.

Nous ferons l'hypothèse que l'emploi des formes déictiques proximales (ici, maintenant, ce $\mathrm{N}$-ci en français, et here, now, this (N) en anglais) exprime l'implication personnelle, subjective de la part du locuteur avec le référent (cf. aussi Béguelin, I998: 95); alors que celui des formes distales (là, alors, ce N-là en français, et there, then, that $(N)$ en anglais) suppose soit la dissociation personnelle, subjective du locuteur par rapport au référent, soit un alignement entre locuteur et allocutaire, l'entité visée étant conçue comme relevant d'une information déjà négociée, en termes interactionnels. En revanche, selon la conception traditionnelle, ce choix serait motivé plutôt par le degré de distance spatiale qui existe entre le référent visé dans la situation d'énonciation et le locuteur (voire l'allocutaire): objet de la référence situé relativement proche du locuteur, vs. relativement éloigné (et/ou relativement près de l'allocutaire), respectivement.

La suite de l'article est organisée de la façon suivante: la section 2 décrit, à partir d'exemples attestés, les marqueurs nominaux français en ce $\mathrm{N}$-ci et ce $\mathrm{N}$-là. La section 3 examine des faits comparables fournis par l'anglais, en analysant des exemples comportant les démonstratifs this $(N)$ et that $(N)$. Une fois ces bases établies, la section 4 compare deux hypothèses opposées sur le fonctionnement de ces formes en discours - l'une reposant sur les projets communicatifs des interlocuteurs, l'autre sur les caractéristiques externes de l'espace perceptif de la situation d'énonciation. Enfin, la section 5 fait le point sur les arguments avancés et les analyses des sections 2 à 4 , et conclut en faveur de la première des deux hypothèses potentiellement explicatives présentées à la section 4 .

2. LES MARQUEURS DÉMONSTRATIFS NOMINAUX FRANÇAIS CE $N-C I / C E N-L \grave{A}$

Il est bien connu que les particules démonstratives françaises -ci et -là sont dérivées respectivement des adverbes démonstratifs spatiaux ici et là (voir Guillot, à paraître, 
20 I5, pour les détails). Le déterminant démonstratif ce a perdu la force déictique que possédaient les démonstratifs en ancien français (voir à ce sujet De Mulder et Carlier, 2006), force que maintiennent depuis l'ancien anglais les formes this (N) et that $(\mathrm{N})$ en anglais moderne.

Par hypothèse, donc, les marqueurs démonstratifs de l'ancien français fonctionnaient à l'époque de façon semblable à l'opposition entre this et that en anglais contemporain. D'ailleurs, De Mulder et al. (2010: 89, n. Io) rapprochent la forme ce $\mathrm{N}$-ci du déterminant néerlandais proximal deze, qui est effectivement luimême très proche du démonstratif proximal this de l'anglais. Ce facteur expliquerait pourquoi, en français moderne, quand le besoin se fait ressentir d'employer déictiquement les déterminants démonstratifs, là où la distinction déictique entre domaines 'proximal' et 'distal' sera pertinente, le locuteur se voit obligé de leur adjoindre l'une ou l'autre des particules démonstratives -ci ou -là.

Cela n'empêche pas, cependant, les SN démonstratifs en ce $N$ d'être employés pour réaliser la deixis en situation. Mais ce serait là une deixis relativement "faible » par rapport à celle exprimée via ce type de SN étendu par l'une ou l'autre des particules démonstratives $-c i$ ou $-l a{ }^{2}$ En effet, le déterminant démonstratif $c e$ du français moderne n'est pas dépourvu d'une part d'anaphoricité (je remercie l'un des relecteurs anonymes d'avoir attiré mon attention sur ce point), ce qui expliquerait selon nous la relative " faiblesse " de ses emplois déictiques.

Selon De Mulder et Carlier (2006), le déterminant démonstratif ce est en voie de grammaticalisation vers le statut d'article défini, ce qui entraîne inévitablement un affaiblissement de son sémantisme. Selon les auteurs (ibid., pp. Io8-9), c'est l'emploi 'mémoriel'3 qui serait la source du passage de la 'définitude pragmatique' à la 'définitude sémantique'; et c'est cette dernière qui caractériserait, selon eux, l'article défini en français contemporain (voir aussi Guillot, à paraitre, 2015: 563). Cet emploi, précisément, est réalisé de façon prototypique par les SN démonstratifs distaux en anglais (that/those $N(s)$ ), et par le membre distal cil de l'ancien français également, d'après les auteurs. Or, ce membre de la dyade est précisément la variante non-marquée de l'opposition, ${ }^{4}$ ce qui motiverait d'autant la transition de son descendant vers un article défini: cf. Lyons (1975) pour des faits comparables de l'anglais. Voir aussi à cet égard l'étude détaillée de l'émergence d'un article défini en finnois à partir d'un démonstratif distal dans Laury (I997).

Cil en ancien français provient tout comme l'article défini en français moderne du pronom démonstratif latin (ecce-)ille; cependant, c'est son homologue proximal cist qui a donné naissance au déterminant démonstratif $c e$.

2 Comparer à cet égard le contraste entre le SN étendu ce mois-ci et l'emploi du SN 'nu' ce mois à sa place dans l'exemple attesté $(4 \mathrm{~b})$ à la toute fin de la sous-section 2.I plus loin.

3 Phénomène que j'appelle 'anadeixis de reconnaissance'. Voir comme illustrations les exemples (5) et (8) infra.

${ }^{4}$ Voir Debruyn (I992: 6), qui se fonde sur les fréquences respectives d'occurrence des deux membres de la dyade ce $N$-ci vs. ce $N$-là, son corpus écrit provenant de Frantext: l'auteur relève $89 \%$ occurrences du second type de marqueur, pour seulement I I \% du premier. 


\section{Francis Cornish}

Comme le soutiennent Kleiber (2008) et Le Draoulec (2013), ici, adverbe démonstratif 'proximal', marqué, ne peut réaliser en contexte que la deixis; là, ${ }^{5}$ par contre, exprime de préférence l'anaphore (où plutôt, dans le cadre de ce travail, l'‘anadeixis' — voir plus bas). En voici un exemple récent:

\section{(I) 'Bruxelles dernière}

PARIS PREMIÈRE

\subsection{0 | magazine |}

Les déambulations nocturnes de Philippe Besson dans la ville belge

... Plus largement, Philippe Besson s'intéresse aux rapports qu'entretiennent les Belges avec leur pays, qui pour certains "n'existe pas ». Là, peut-être, réside la clé de cette liberté débonnaire, naturelle et loufoque qui semble régner sur les nuits bruxelloises.' (Christine Rousseau, Le Monde Supplément Télévisions, 26-27/05/I 3 , p. I4)

Dans cet exemple, l'adverbe distal là à la ligne 2 du corps du texte est en position focale, suivie d'une séquence verbe-sujet postposé. À ce titre, sa fonction anadéictique (et non purement anaphorique, au sens strict) est en exergue: il assume en effet une fonction anadéictique et non anaphorique au sens strict. Comme ce marqueur réalise une fonction focale dans l'énoncé, il serait accentué et recevrait un accent de hauteur à l'oral. Il fonctionne donc pour partie déictiquement, servant à pointer avec une certaine force vers la représentation discursive que la prédication indexicale hôte permet de dégager du contexte en amont. Il s'agit ainsi d'un renvoi quasi "déictique de discours " (voir plus bas) à un objet de discours caractérisable comme 'le fait que pour certains Belges, la Belgique en tant que pays unitaire n'existe pas'. La notion d'،anadeixis' sera présentée plus bas.

La distinction relative entre fonction déictique pure (ici) et anadéictique (là) se reflète dans l'Echelle des marqueurs indexicaux que j'ai proposée (Figure I plus bas), de par la position plus proche du pôle déictique adoptée par ici, là se situant plus près du pôle anaphorique — mais toujours au sein de l'empan 'Anadeixis' dans l'échelle. La même configuration caractérise les SN démonstratifs à particule démonstrative ce $N$-ci et ce N-là. Par 'indexical', nous entendons le fait de 'pointer' par le biais du contexte de l'occurrence de la forme employée.

Nous adoptons ici une conception 'scalaire' de la relation entre deixis et anaphore, en nous inspirant de sa caractérisation par Lyons (I975) et par Bühler (2009)/[I934]. Il y aurait ainsi un continuum d'indexicalité allant de la deixis (procédure indexicale fondamentale, première) à l'anaphore (procédure indexicale dépendante, dérivée), en passant par une zone intermédiaire appelée 'Anadeixis' (terme d'Ehlich, I982). La Figure I ci-dessous reflète ce continuum.

La Figure I donc range ro grandes catégories de marqueurs indexicaux sous forme d'une échelle de leurs propriétés indexicales intrinsèques. ${ }^{6}$

${ }^{5}$ Voir aussi l'analyse des emplois discursifs plus étendus de ce dernier marqueur que propose Forget (I989).

${ }^{6}$ Abréviations employées dans la Figure $\mathrm{I}$ : ' $\mathrm{pI} \mathrm{I}^{\text {èe }} / 2^{\text {ème }} / 3^{\text {ème }} \mathrm{p}$ ': 'pronom de première/deuxième/troisième personne'; 'P': 'proximal'; ' $\mathrm{D}$ ': 'distal'; 'dm': 'démonstratif'; 


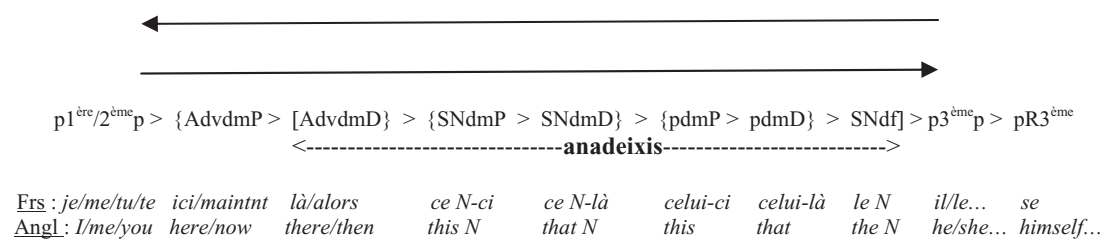

Figure I: Echelle d'indexicalité encodée par certaines catégories d'expressions indexicales (Cornish, 2010b, Figure 1, p. 116 -révisée). Les accolades renferment les membres de certaines micro-oppositions formées par des paires de marqueurs.

La raison d'être de cette échelle réside dans le degré d'indexicalité inhérent à chaque catégorie indexicale retenue. Ce qui motive le placement des catégories et de leurs marqueurs caractéristiques sur l'Échelle est non seulement leur fréquence relative d'occurrence dans la réalisation des diverses procédures indexicales référentielles, telles qu'elles se manifestent dans les corpus, mais aussi, et surtout, leurs propriétés morpho-syntaxiques, sémantiques et pragmatico-discursives qui les rendent susceptibles de le faire.

Je précise que ce sont les paires de marqueurs adjacents sur l'échelle (je/tu, ici/là, maintenant/alors, ce $\mathrm{N}$-ci/ce N-là, celui-ci/celui-là), qui constituent des microoppositions unitaires, qui doivent être comprises comme entrant dans la succession sérielle sur l'échelle, et non les marqueurs pris individuellement (à l'exception bien entendu des marqueurs qui n'entrent dans aucune micro-opposition de cette sorte : à savoir, les $\mathrm{SN}$ définis, les pronoms de $3^{\text {ème }}$ personne, puis les pronoms réfléchis de $3^{\text {ème }}$ personne). Mais au sein de chaque paire, les membres des micro-oppositions concernées sont ordonnés en fonction de l'échelle d'indexicalité.

Les deux pôles sont occupés, respectivement, par les pronoms personnels de $\mathrm{I}^{\text {ère }}$ et de $2^{\text {ème }}$ personne, qui sont intrinsèquement déictiques (fonctionnant de manière 'token-reflexive') et qui ne peuvent s'employer anaphoriquement; puis par les pronoms réfléchis clitiques (en français) de $3{ }^{\text {ème }}$ personne, qui de façon prototypique ne fonctionnent qu'anaphoriquement en termes de liage, et de plus à l'intérieur d'une proposition. Leur champ d'opération est de ce fait fortement contraint. On notera par ailleurs que la référence de ces deux types de marqueurs

'adv': 'adverbe'; 'SN': 'syntagme nominal'; 'p': 'pronom'; 'df: 'défini'; 'R': 'réfléchi'. Notons que la raison d'être de la série de marqueurs en anglais et en français placés chacun au-dessous de chaque catégorie indexicale dans l'Échelle est uniquement d'illustrer chacune de celles-ci: la relation cruciale existant entre chacun de ces marqueurs (et la catégorie indexicale qu'il illustre) est uniquement d'ordre intra-langagier (à lire donc 'horizontalement'). Leurs rapports ne devront donc pas se lire 'verticalement', donc interlangagièrement. En effet, il y a des différences significatives, par exemple, entre les SN définis en français et en anglais, de même qu'entre les pronoms réfléchis de zème personne en français (qui sont des clitiques) et en anglais (qui sont des expressions nominales, potentiellement argumentales, susceptibles d'être accentuées). 


\section{Francis Cornish}

polaires se réalise quasi automatiquement, sans que l'interprète soit obligé de passer par un processus de résolution via le contexte plus large.

Au milieu de l'échelle se trouve tout un empan marqué 'Anadeixis'. L'anadeixis' correspond au mode de référence indexicale qui combine les procédures anaphorique et déictique à des degrés différents. Il y a selon moi trois sous-types essentiels d' 'anadeixis', que l'on peut ranger par ordre croissant (allant de droite à gauche) sur l'axe "Deixis-Anaphore " proposé pour l'ensemble de marqueurs indexicaux dans la Figure I plus haut:

- l'anadeixis 'stricte' (cf. le terme 'démonstratifs anaphoriques' utilisé par Kleiber, I990 et Diessel, I999): le renvoi à un référent qui peut avoir été évoqué antérieurement dans un discours, mais qui n'est plus — ou n'est pas encore - topical au moment de ce renvoi: le référent ainsi ciblé existe bel et bien dans le discours environnant, mais il est d'un accès relativement difficile — d'où l'implication de la dimension déictique. Voir les exemples français (2) et (3) plus loin dans cette même section;

- l'anadeixis de reconnaissance/de rappel (cf. la notion de 'deixis mémorielle'): le pointage vers une entité — souvent un événement, parfois stéréotypique — présumée partagée au sein de la mémoire à long terme des participants: ici, le référent ciblé existe également indépendamment de cette référence indexicale; mais il est d'un accès encore plus difficile, étant localisé dans la mémoire à long terme des participants — d'où la primauté de la dimension déictique dans de tels renvois. Voir l'exemple français (5) plus loin; et

- la deixis de discours: le pointage cognitif vers une représentation discursive en mémoire de travail, et la création par inférence à partir de celle-ci d'une entité discursive pour partie nouvelle: ici, le référent impliqué n'existe même pas en tant que tel avant l'acte de référence indexicale; la dimension déictique remplit de ce fait un rôle encore plus appuyé dans ce type de référence —d'où le nom de cette sous-procédure indexicale. Voir comme illustrations les exemples (6) et (7) dans cette même section.

Concernant l'empan de l'Echelle marqué 'Anadeixis', donc, il s'agit pour la plupart de types d'expressions à base démonstrative rangés entre les deux catégories polaires. Ces expressions démonstratives (adverbes déictiques, SN et pronoms) sont ordonnées en fonction de la distinction proximal (marquée) vs. distal (non marquée) qu'elles peuvent exprimer morphologiquement - la variante marquée possédant un degré plus élevé de déicticité que la variante non marquée (cf. Langacker, 2002b: 34; Lyons, 1975).

Les adverbes déictiques (par ex. maintenant/alors, ici/là en français) sont placés à une position plus élevée que les SN lexicaux: voir Himmelmann (I996: $245-246$, n. I2) pour des indices trans-langagiers selon lesquels les déterminants et pronoms démonstratifs sont souvent dérivés au plan diachronique à partir d'adverbes démonstratifs, qui sont des types d'expression plus fondamentaux — au sens où ils existent à des états antérieurs des langues en question par rapport à ceux-là. En outre, selon l'auteur, leur fréquence dans les corpus (narratifs) serait plus élevée.

${ }^{7}$ Voir de Mulder et Vetters (2008) pour l'adverbe temporel maintenant, et Kleiber (2008) sur ici et là. Pour les premiers, l'adverbe proximal maintenant ne réalise que la deixis, et son homologue temporel distal, alors, fonctionne de préférence anaphoriquement (ou plutôt, comme nous le dirions, 'anadéictiquement'). Pour Kleiber, comme nous l'avons déjà indiqué, il en est de même pour la paire d'adverbes spatiaux ici (proximal) et là (distal). Pour ce qui concerne l'anglais here et now, voir Ariel (I998), et pour then, Schiffrin (I990). 
Enfin, quant aux SN lexicaux, ceox-ci se trouvent à une position plus haute dans l'Échelle que les pronoms correspondants.

Toutes les catégories à base démonstrative sont placées au-dessus de la catégorie des SN définis sur cette Echelle: j’ai situé les SN définis à la limite inférieure de l'empan 'anadéictique' dans la Figure I, car même s'ils ne réalisent pas toujours une fonction indexicale, ${ }^{8}$ ils peuvent néanmoins connaître des emplois déictiques, anadéictiques 'stricts' ou anaphoriques. Ce cas montre bien la flexibilité indexicale des marqueurs au fur et à mesure que l'on descend l'Échelle: par exemple, ce $\mathrm{N}$-ci peut bien réaliser un acte de référence déictique pur (comme illustré dans les exemples (4a) et (4b) plus bas), de même que son homologue pronominal celuici. Cependant, il fonctionne également très naturellement de manière anadéictique (voir les exemples (2) et (3) dans la sous-section 2. I infra). Il n'est donc pas cantonné à n'exprimer que la deixis. Voir Cornish (20II) pour une discussion plus ample portant sur les faits de l'anglais.

L'échelle reconnait des positions relatives, et non 'absolues', des catégories de marqueurs retenues. Il s'agit de ce que la langue-système rend disponible aux usagers pour référer indexicalement. Dans l'emploi en contexte, plusieurs facteurs peuvent intervenir pour moduler les valeurs systémiques que reflète cette échelle: la nature du nom dans le cas des marqueurs nominaux (SN), le cotexte environnant, la prosodie, la structure informationnelle, et d'autres facteurs contextuels encore. Mais ces facteurs ne sont pas à même de neutraliser l'apport de la distinction démonstrative entre membres proximal et distal, pour ne prendre que cette opposition marquée morphologiquement, et donc leurs positions relatives sur l'Echelle des marqueurs indexicaux. ${ }^{9}$

On notera à ce sujet la discussion détaillée de Debruyn (I992) sur l'apport de quatre types de noms têtes au sein de la paire de marqueurs démonstratifs ce $\mathrm{N}$-ci et ce $N$-là: à savoir, les noms dénotant les objets concrets, abstraits, temporels et spatiaux. Selon Debruyn, il y aurait deux modèles de fonctionnement de cette paire: dans le cas du choix d'un nom tête désignant un objet concret, abstrait ou spatial, les deux membres seraient dans un rapport d'opposition l'un à l'autre. Ce N-ci serait contraint de renvoyer à ce qui est dans le focus ou foyer d'attention au moment de son emploi, alors que ce $\mathrm{N}$-là ne connaitrait pas une telle restriction. Comme

${ }^{8}$ En effet, ils peuvent référer indépendamment en fonction de leur contenu lexical, lorsque celui-ci suffit à identifier leur référent de manière non-équivoque.

9 À l'exception, toutefois, de la prosodie, pour ce qui concerne les pronoms anglais: en effet, un accent de hauteur sur ces pronoms (HE vs. he par exemple) assigne au marqueur ainsi doté un caractère anadéictique, le plaçant de cette façon à une position plus élevée vers le pôle déictique par rapport à son homologue inaccentué. Mais ce dernier a uniquement une vocation anaphorique, au sens strict. Cependant, il y a de bonnes raisons de considérer que les pronoms accentués constituent des marqueurs indépendants, à part entière: après tout, même s'ils ne different pas morphologiquement de leurs homologues inaccentués, ils possèdent tout de même des propriétés phonologiques et discursivo-pragmatiques distinctes. À ce titre donc, ils se placeraient de façon 'permanente' sur l'Echelle des marqueurs indexicaux à une position plus proche du pôle déictique que les pronoms de $3^{\text {ème }}$ personne inaccentués. 


\section{Francis Cornish}

il a été proposé par De Mulder et al. (2010) par rapport à la situation en français médiéval, ce $\mathrm{N}$-là trouverait son référent en dehors des circonstances immédiates de l'acte d'énonciation.

Par contre, lorsque le nom tête de ces marqueurs a une valeur temporelle, il existerait un rapport d'inclusion entre le moment ou la durée désignés par ce $\mathrm{N}$-ci et le moment de l'acte d'énonciation (cette forme serait donc contrainte à ne réaliser que la deixis); alors que l'emploi de ce $N$-là avec un tel nom tête, lui, serait régi par une contrainte d'exclusion par rapport à la situation d'énonciation. Sa 'saturation' référentielle serait de ce fait effectuée par le truchement du contexte discursif plus large: un fonctionnement anaphorique donc, ou plutôt, comme nous le dirons dans ce cas, 'anadéictique'. Nous verrons des indices de ce genre plus loin, en analysant nos propres exemples.

\section{$2.1 \mathrm{Ce} N-c i$}

Voici à présent un premier exemple attesté de l'emploi de ce $N$-ci, membre marqué de l'opposition ce $\mathrm{N}$-ci/ce $\mathrm{N}$-là.

(2) '... La question, ${ }^{10}$ pourtant, n'est pas définitivement tranchée dans mon esprit. Car la position « républicaine » ne me semble pas seulement inspirée par la nécessité de répondre à la menace islamiste, mais par la constante difficulté de la politique française à faire place à la diversité. Ce que montre assez le problème, si étrangement dramatisé, des langues régionales, avec sa récente relance. Dans ce débat-ci, je sais mieux de quel côté je penche. . ' (M. Ozouf, 2009, Composition française, Gallimard, pp. 266-7)

La notion de 'débat' n'a pas été explicitement mentionnée dans le cotexte précédant cet extrait; ${ }^{11}$ cependant, c'est cette re-catégorisation de la question controversée des langues régionales qui oppose bon nombre de concitoyens ces derniers temps, que propose l'auteur. Elle indique clairement où elle se situe au sujet de ces controverses, donc la question du port du foulard dans les écoles et celle de la diversité — représentée ici par le statut des langues régionales - vs. l'universalité, présentées comme en opposition l'une par rapport à l'autre: à témoin le connecteur adversatif mais à la ligne 3 de l'extrait. En employant le SN démonstratif marqué ce débat-ci tout de suite après la mention de la question controversée de la diversité, et en particulier en ce qui concerne le statut des langues régionales à côté du français, langue officielle, l'auteur exploite la procédure de la deixis —en partant de l'occurrence même du marqueur proximal pour rehausser la saillance de ce qui vient d'être mentionné, et en l'érigeant en topique par opposition à son concurrent, la question du port du foulard dans les écoles.

$\mathrm{Au}$ terme de leur étude de l'opposition démonstrative entre ce $\mathrm{N}$-ci et ce $\mathrm{N}$ là en moyen français, De Mulder et al. (20IO: IOO) soutiennent que la particule suffixale $-c i$ 'signale que le référent doit être identifié par le truchement du contexte

${ }^{10}$ Celle de l'interdiction du port du foulard islamique à l'école en France.

${ }^{11}$ Notons l'emploi des noms généraux question (ligne I) et problème (ligne 4) dans le co-texte précédent pour caractériser ces référents abstraits. 
d'énonciation, comportant le locuteur, le moment et le lieu d'énonciation, et le contexte qui accompagne l'occurrence démonstrative'. Nous pourrions interpréter cette description comme signifiant que l'emploi des formes ainsi constituées correspond à l'expression de la deixis pure. Toutefois il s'agit plutôt ici de références de type 'anadéictique' que de 'deixis pure', en réalité — du moins en ce qui concerne la langue actuelle.

En effet dans (2), il y a évidemment aussi une part (relativement ténue) d'anaphoricité dans le renvoi démonstratif, puisque l'objet de discours à l'œuvre vient tout juste d'être évoqué. L'emploi de la particule -ci en (2) exclut par opposition l'autre 'débat' dont il a été question, celui sur le port du foulard islamique dans les écoles. L'emploi à sa place du membre distal de l'opposition (ce débat-là) n'aurait pas eu la même valeur discursive, ni d'ailleurs le même référent.

De plus, nous pouvons postuler, contra Marchello-Nizia (2006: I25), que plutôt que d'avoir un simple 'sens spatial' en français contemporain, les formes démonstratives qui marquent la distinction 'proximal' vs. 'distal' (comme ce débat-ci en (2)) encodent toujours, respectivement, la distinction 'à l'intérieur de la sphère subjective du locuteur'/ 'en dehors de la sphère subjective du locuteur': cf. aussi Guillot (20IO) pour le français médiéval. Voir la section 4 pour une discussion plus approfondie de cette question. En effet, en (2), l'emploi de la variante proximale ce débat-ci manifeste fortement l'intérêt du scripteur pour l'objet de discours ainsi pointé, comme en témoigne la subjectivité de ce qui est prédiqué de ce référent dans la principale : ... je sais mieux de quel côté je penche.

Un autre exemple de ce marqueur se trouve dans (3):

(3) [Article 'Jean-Luc Hees : «Radio France va lancer une plateforme musicale gratuite ».' Interview de Jean-Luc Hees par Daniel Psenny (Le Monde, Supplément Radio-Télévision, 27-28/04/I3, p. 6)]

\section{Le dernier sondage de Médiamétrie, publié à la mi-avril, montre un fléchissement d'audience pour France Inter. Comment l'expliquez- vous?}

France Inter a démarré la saison normalement, avec de bons chiffres à chaque sondage. Mais cette fois-ci, la station a connu six jours de grève qui nous ont fait perdre un million d'auditeurs...'

Ici, il s'agit d'un contraste entre deux moments dans la popularité de la station de radio publique France Inter: le début de la saison en cours à l'époque (la rentrée de septembre 20I2), d'une part, et la situation à la mi-avril 20I3 au moment de la publication du dernier sondage d'audience de la station, de l'autre. Il y a une part, certes mince, d'anaphoricité dans cette référence (manifestée entre autres lexicalement de par le choix du nom fois comme tête de ce $\mathrm{SN}$ ), ce qui justifierait la qualification de référence 'anadéictique' ici - malgré le fait que ce nom ne soit pas apparu en tant que tel précédemment. Cependant, selon Debruyn (I992: 32-33), comme nous l'avons vu, lorsque le $\mathrm{N}$ tête du marqueur proximal ce $\mathrm{N}$-ci dénote un moment ou une durée, comme dans (3), alors il est interprété en rapport avec 'l'environnement temporel de l'énoncé' (p. 32). Il y aurait donc dans ce cas 
un rapport 'inclusif', selon Debruyn, entre le temps de l'énonciation et le temps désigné par le marqueur (il s'agirait de ce fait d'un emploi (ana)déictique).

Pour Theissen (2008), le nom tête fois dans de tels marqueurs ne se comporte pas comme les noms têtes des SN démonstratifs en ce $N(-c i /-l a ̀)$ dénotant une entité de premier ordre (un objet spatio-temporellement délimité). Car sa présence en crée non une expression argumentale, mais un adverbial de phrase, cadratif (d'où sa préférence pour la position initiale, comme dans (3)). Le nom fois ici fonctionnerait comme une sorte d'opérateur, exigeant d'être interprété en termes d'une pluralité d'occurrences d'un événement en série (p. 213). C'est bien le cas dans (3), où le démarrage "normal " de la saison par France Inter constituerait l'événement " antécédent ", et les six jours de grève puis le fléchissement de popularité à la miavril $20 \mathrm{I} 3$ correspondrait à la «nouvelle " 'fois', ce qui créerait donc une mini-série d' « occurrences processives ».

À noter aussi la présence du connecteur adversatif mais dans l'extrait (3) reliant les deux segments de texte qui évoquent chacun un terme de l'opposition. L'emploi de cette fois-ci s'expliquerait par ce contraste voulu entre les 'bons chiffres' de l'avantdernier sondage et la baisse d'audience détectée par le tout dernier (et fort récent, au moment de cette interview et sa publication). Voir aussi Theissen (2008: 222-223) sur l'effet contrastif associé à l'emploi de cette fois(-ci/-là).

Un exemple de deixis spatiale pure réalisée par ce $\mathrm{N}$-ci est présenté en (4a), puis de deixis temporelle en $(4 \mathrm{~b})$ :

(4) a. 'Si tu ne vas pas à Yokohama, a dit Hélène, tu n'as qu'à décrire cette chambre-ci à la place. On peut le faire là, maintenant, ça nous occupera.' (E. Carrère, D’autres vies que la mienne, cité par Le Draoulec, 20 I3, ex. (I 5), p. 92)

Le démonstratif cette chambre-ci renvoie directement à la chambre dans laquelle se trouvent les interlocuteurs au moment de la parole (au $28^{\text {ème }}$ étage de l'hôtel Intercontinental à Hong Kong). Il s'agit donc d'un cas de deixis pure, et non d'anadeixis, comme dans les deux exemples précédents.

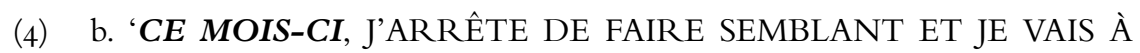
LA SOIRÉE DES VIDÉOPHAGES' (plaquette, 'Projection de courtsmétrages indépendants', lundi 6 janvier 20 I4-20h, salle San Subra, 2 Rue San Subra - quartier Saint Cyprien - Toulouse)

Le SN proximal ce mois-ci ici renvoie déictiquement au mois en cours au moment de la parole énoncée (janvier de l'année en question). Il s'agit donc également d'une référence déictique " pure».

\section{$2.2 \mathrm{Ce}$ N-là}

Observons maintenant quelques emplois attestés du SN démonstratif distal ce N-là:

(5) [Photo en noir et blanc d'un cercueil drapé d'un linceul, sur lequel est posée une rose (Publicité pour les Pompes Funèbres Générales, Le Monde 05/ I I/93):] Légende: 'Notre métier est de vous aider dans ces moments-là' 
Il n'y a pas d' 'antécédent' textuel ici, le SN indexical ces moments-là renvoyant à la représentation (stéréotypique) supposée partagée en mémoire à long terme, 'les obsèques d'un être cher'. Le nom tête au pluriel moments fournit (de façon très générale) la catégorie d'entité à retirer de la mémoire à long terme. ${ }^{12}$ Le nombre pluriel porté par ce SN ainsi que le choix du lexème-tête moments (fortement hyperonymique) pourraient relever d'un euphémisme de la part du scripteur, évitant ainsi la focalisation sur le type d'événement (pénible) évoqué qui correspondrait au choix du nombre singulier — de par le renvoi à l'ensemble de tels 'moments'. Il est clairement question ici, selon moi, d'une référence 'anadéictique' (du type 'anadeixis de reconnaissance'), où la dimension 'anaphorique' est très bien représentée - de façon plus substantielle encore que dans le cas de l'emploi de son homologue proximal ce débat-ci dans (2) ou de cette fois-ci dans (3): car l'existence du stéréotype des obsèques d'un être cher est bien entendu fortement présupposée.

Cela est conforme à ce que postulent De Mulder et al. (20 IO: IOO) pour le français médiéval, lorsqu'il s'agit de la particule suffixée -là: à savoir que la présence de ce suffixe 'signale que le référent doit être identifié par le truchement d'éléments qui ne font pas partie de son contexte d'énonciation' (ici donc le renvoi au stéréotype des obsèques). ${ }^{13}$ Cette idée est confirmée par l'analyse de Debruyn (I992) de ce type de marqueur, quand la tête dénote une entité temporelle: selon lui, également, dans ce cas la saturation d'une telle forme devra se faire en détachement des circonstances temporelles de son énonciation, ce qui est bien le cas dans (5). Cependant, tout comme l'emploi du suffixe -ci, le mode de fonctionnement de -là n'en est pas moins 'token-réflexif, selon nous.

La forme ces moments-là dans (5) combine de ce fait référence anti-déictique (pointage en fonction de l'acte même d'énonciation de ce marqueur vers la représentation mémorielle partagée stéréotypique qui, elle, ne fait pas partie des circonstances immédiates de son énonciation) et référence anaphorique - d'où la qualification d' 'anadéictique': à ce titre, le texte de la légende fournit un commentaire sur cette situation, supposée à présent familière au lecteur à travers la photo: c'est cette valeur que la particule démonstrative suffixale -là contribue à la référence du SN dans son ensemble. Si on remplaçait la particule -là ici par $-c i$, cette valeur ne serait absolument pas disponible, car le lecteur serait orienté ainsi de façon insistante vers la situation évoquée dans l'image en tant que telle. Il n'y aurait pas renvoi au stéréotype des obsèques représenté en mémoire à long terme, et la valeur généralisante serait également perdue (ou serait du moins amoindrie).

${ }^{12}$ Cependant, il incombera évidemment à l'interprète d'inférer, à partir de l'image conjointement à sa légende, la nature des 'moments' visés ici. Ce facteur correspondrait parfaitement à la caractérisation par De Mulder et Carlier (2006: I09) de cet emploi de la forme distale, que l'on reverra plus bas.

${ }^{13}$ On pourrait même appeler ce fonctionnement 'anti-déictique', par opposition à celui de son homologue proximal: 'anti-déictique' au sens où le fonctionnement du marqueur serait néanmoins token-réflexif; mais il comporterait aussi une instruction tacite à l'interprète de chercher son interprétation ailleurs que dans les circonstances immédiates de l'énonciation. 


\section{Francis Cornish}

Voici maintenant un autre exemple attesté de ce marqueur:

(6) ‘. . Le profil des auteurs des attentats de Boston a mis en lumière une étude récente publiée par la Rand Corporation, un organisme proche des services de sécurité, selon laquelle, sur les Io4 projets d'attentat recensés aux EtatsUnis depuis 200I, les trois quarts impliquaient des citoyens américains. Ce terroriste-là n'aurait plus besoin de partir s'entrainer au djihad dans les zones tribales du Pakistan. Eduqué, il suffirait au nouveau " terroriste Internet " de suivre les prêches salafistes sur la Toile, où il acquiert aussi le bagage nécessaire à la menée d'opérations qui peuvent aussi bien être organisées de l'extérieur que le résultat d'initiatives isolées.' (Le Monde 2 I-22/o4/I 3, p. 2)

Ici, l'emploi du SN démonstratif distal ce terroriste-là (ligne 4) catégorise l'auteurtype des attentats commis ou à commettre sur le sol américain comme 'terroriste', mais l'érige en un type particulier de par le choix du nombre singulier et non pluriel - c'est donc quasiment un cas de la procédure 'deixis de discours' ici (voir à cet égard l'exemple anglais (9) au $\S 3$ plus bas). Il s'agit, non pas d'une référence quasi 'générique' aux responsables effectifs des Io4 'projets d'attentats' à l'intérieur des Etats-Unis recensés depuis 200I, mais au profil du (nouveau type de) terroriste en question: un 'citoyen américain', opérant donc à l'intérieur du pays où il réside lui-même, et non un étranger venant du dehors. Cette dernière conception correspondrait à l'idée commune que se faisaient les Américains depuis les événements tragiques du I I septembre 200 I. La notion de 'profil' des terroristes est d'ailleurs rendue explicite dans la première phrase de l'extrait, ce qui permet d'anticiper ce renvoi indexical dans la seconde.

Il ne s'agit pas non plus d'une reprise du seul suspect présumé responsable de l'attentat du marathon de Boston le i9 avril 20I3 encore en vie au moment de la publication du journal source (à savoir, Djokhar Tsarnaev). Or, même si la caractérisation de ce 'nouveau' type de terroriste vient d'être évoquée, c'est l'emploi du SN à particule démonstrative distale et non proximale (comme en (2) et (3)) qui est favorisé ici. Cet emploi ne pourrait donc pas relever d'un simple ordre d'occurrence relative du segment textuel évoquant le référent visé, comme le voudrait la description traditionnelle: voir la section 4 pour une discussion plus ample à cet égard. L'emploi potentiel du marqueur ce(s) terroriste(s)-là pourrait renvoyer au profil des deux terroristes mentionnés dans la première phrase de l'extrait, et ce terroriste-ci, lui, reprendrait le nouveau type de terroriste états-unien qui vient d'être évoqué - mais selon moi, la variante proximale ce terroriste-ci parait moins naturelle dans ce contexte.

Cependant, le contenu prédicatif de la phrase hôte dans (6) n'est compatible qu'avec le second de ces deux renvois potentiels en tant qu'il sera réalisé par le marqueur distal ce terroriste-là. Selon nous, ce choix relève de la construction d'un 'type' d'entité, un peu comme dans le cas de l'emploi du même type de marqueur en (5): mais là il s'agissait de renvoyer à un stéréotype supposé déjà installé dans la mémoire à long terme du lecteur. 
Voici à présent un deuxième exemple de ce type d'emploi de ce $N$-là, qui fonctionne anadéictiquement en construisant à part entière un objet de discours qui vient d'être esquissé, en le classifiant comme entité d'un certain type:

(7) 'Déshabillons-les

PUBLIC SÉNAT

23.45 | MAGAZINE |

Comment les humoristes au pouvoir caricaturent-ils François Hollande?

Les humoristes l'imitent ou le moquent en faisant de lui un personnage à la fois mou, craintif, sans relief et légèrement benêt. Des "Guignols " à Nicolas Canteloup, en passant par Laurent Gerra et Didier Porte, tous dépeignent François Hollande comme un président peu charismatique, incapable d'arbitrer et d'agir.

"On s'engouffre tous dans la brèche une fois que c'est admis par l'opinion. On est, nous les humoristes, souvent obligés d'aller dans ce registre-là, explique Didier Porte, avant d'ajouter : Mais on peut aussi montrer qu'on n'est pas dupes, se démarquer. Parce que moi, sincèrement, je trouve le type pas du tout mou. Je trouve qu'il a le cuir tanné et qu'il a les nerfs sacrément solides, autrement plus solides que ceux de son prédécesseur. ( . . . ) ».' Hélène Delye, Le Monde Supplément Radio-Télévision, 27-28/04/I3, p. I4

Ici, il y a contraste ${ }^{14}$ entre la caractérisation 'caricaturale' de François Hollande présentée comme largement adoptée actuellement par les humoristes ainsi que par l'opinion en France (voir le propos du premier paragraphe de l'extrait), et celle plus sobre et positive prônée par Didier Porte. Cette dernière, que DP prend à son compte, est empreinte de subjectivité: témoin les expressions 'on n'est pas dupes', 'moi, sincèrement... ', 'le type', 'il a le cuir tanné' et 'les nerfs sacrément solides'. Tout comme dans les exemples (2) et (3), nous remarquons ici l'emploi du connecteur adversatif mais pour distinguer les deux termes de l'opposition, à la ligne 7 .

Comme dans le cas du démonstratif distal anglais that (cf. Cornish, 200I), nous pouvons dire qu'il s'agit ici d'un emploi 'modal' de ce membre de la dyade démonstrative, le locuteur se dissociant de la perception communément admise (à l'époque) du Président français, de par son choix du membre distal, non marqué, de la paire: voir le $\S 4$ pour une discussion en termes plus généraux. Comme dans le cas du marqueur ce terroriste-là dans (6), ce n'est pas le membre proximal qui est employé à ce point du discours — même si les ingrédients de l'objet de discours ainsi créé ont été évoqués très récemment dans le fil du texte. Il s'agit donc aussi, comme dans (6), de la création (dans la perspective du lecteur) d'un objet de discours ayant la propriété distinctive d'être du type 'registre'. A ce titre, nous pourrons postuler qu'il s'agit même d'un cas de 'deixis de discours', et non d'une simple occurrence d' 'anadeixis «stricte"'.

Notons que, mis à part sans doute celui du SN démonstratif complexe ce terroristelà en (6) qui correspond à une classification plutôt objective du référent visé, les noms têtes des marqueurs que nous avons vus dans cette section représentent tous

${ }^{14}$ Cf. la remarque de Forget (I989: 58) sur la valeur contrastive que peut avoir l'adverbe/suffixe (-)là. 
des classifications revêtant une part plus ou moins grande de subjectivité associée aux référents ciblés de la part des scripteurs ou du locuteur concerné(s).

\section{LeS Marqueurs démonstratifs NominauX ANglais this}

(N) VS. THAT (N)

Pour ce qui concerne les marqueurs anglais équivalents (this $N$ vs. that $N$ ), il est clair tout d'abord que, si l'on substituait la variante proximale à son homologue distal that quietly spoken voice (ligne 5) dans l'exemple (8), l'effet produit serait radical:

(8) 'Originally written and read by their author Alan Bennett ( . . ) for TV, these autobiographical stories work so much better on the radio. He re-recorded them for Radio 4 in just one day back in 2000 with the then producer and now head of programmes at BBC 7 , Mary Kalemkerian. These snapshots of his childhood growing up in Leeds are delivered in that (/\#this/\#the) quietly spoken voice, where his pauses are just as powerful as the words that led up to or followed them...' ('Choices. Telling Tales' (Mon-Fri I I.30 a.m./ I 2 midnight BBC 7), Radio Times 29.07-4.08.06, p. I26)

Il s'agit ici, comme en (5), de la procédure 'anadeixis de reconnaissance', le SN démonstratif distal that quietly spoken voice à la ligne 5 orientant le lecteur vers une représentation présumée partagée en mémoire épisodique ${ }^{15}$ ("la voix douce caractéristique du dramaturge Alan Bennett'). Or, si on remplace le déterminant that ici par son homologue proximal this (this quietly spoken voice), il ne pourra plus s'agir d' 'anadeixis de reconnaissance'; on aurait plutôt affaire à l'emploi 'indéfini', introducteur d'un topique nouveau, qu'on voit dans l'exemple suivant: ${ }^{16}$

(9) '. . Because I've spent the last few months putting together the new show, I've been soaking up a lot of news. Most of that has been from newspapers, but I try not to miss The World at One on Radio 4. Nick Clarke ${ }^{17}$ has this (/\#that/\#the) deliberately non-combative approach that allows the guests to trip themselves up...' ('What I'm watching'. Armando Iannucci, Radio Times 29.07-4.08.06, p. 3I)

Ici, il s'agit d'une présentation quasiment par 'monstration' (comme dans le cas de la deixis canonique, situationnelle) d'un référent tout nouveau pour le lecteur, caractérisé par un SN démonstratif proximal dont la composante lexicale est complexe et étendue (voir le SN démonstratiflignes 3-4). C'est un cas de deixis de discours selon moi, le référent étant créé discursivement de toutes pièces; en outre, il se détache de son contexte (ce qui est le propre de tout acte de référence déictique selon Kleiber, I994) et est de ce fait même rendu saillant. Selon Himmelmann (I996: 207), en revanche, ce genre d'emploi constituerait un sous-type de deixis situationnelle, canonique. Pour nous, cependant, l'effet de l'emploi du déterminant

\footnotetext{
${ }^{15}$ C'est-à-dire, la mémoire d'événements ou de faits ponctuels en tant que tels.

${ }^{16}$ Voir Prince (I98I) pour une étude approfondie de ce type d'emploi du démonstratif proximal this $N$.

${ }^{17}$ Nick Clarke était à l'époque l'animateur de cette émission radiophonique.
} 
démonstratif proximal en (9) est d'ouvrir un 'créneau' discursif qui est marqué comme devant être élaboré immédiatement par des précisions sur l'entité introduite dans le discours, entité à laquelle le locuteur attribue une certaine importance.

Cependant, pour revenir brièvement à l'exemple (8), le contexte à l'œuvre ne permettrait pas l'expression de cette valeur par le choix de la variante proximale du déterminant démonstratif, qui serait plus nettement déictique que ne l'est celle associée à l'emploi de la variante distale — qui, lui, comprend une part bien plus importante d'anaphoricité. En effet, l'emploi de cette forme aurait pour effet de rendre emphatique l'information sur la qualité de la voix d'Alan Bennett. La forme acquerrait ainsi une information discursivement nouvelle, l'auteur la prenant subjectivement à son compte - mais une telle interprétation serait incompatible avec le contexte de l'expression en (8), stylistiquement comme communicativement. Ces valeurs différentielles sont conformes aux positions relatives de ces deux membres de la paire des démonstratifs sur l'Échelle des marqueurs indexicaux (Figure I).

Tournons-nous à présent vers l'un des deux autres SN démonstratifs employés en (8), à savoir These snapshots of his childhood growing up in Leeds (ligne 4). Ce SN démonstratif proximal, bien que fonctionnant de manière 'anadéictique' ('stricte'), comporte un déterminant démonstratif proximal et non l'article défini: cela s'explique sans doute en partie par le fait que le contenu de ce SN ne peut pas être supposé connu au préalable (donc présupposé) par le lecteur de ce texte au moment de l'occurrence. On notera à ce titre le choix par le scripteur du nom tête métaphorique snapshots ('photos'), qui est relativement inattendu comme classification de l'objet (purement auditif) ré-évoqué ainsi.

Cette (re-)classification propre à l'emploi des SN démonstratifs relève selon nous de la forte charge de subjectivité liée à l'emploi de la procédure (ana)déictique en général. ${ }^{18}$ Le contenu de ce SN démonstratif proximal est de ce fait présenté comme un élément d'information nouveau pour le discours ('discourse-new') - bien que le référent du marqueur dans son ensemble, lui, soit discursivement 'ancien'. Un autre facteur déterminant est le fait que l'auteur de ce texte souhaite à l'évidence maintenir le référent visé au centre de son attention ainsi qu'à celui de son lecteur, en en rehaussant la saillance, comme dans le cas de l'emploi du SN démonstratif proximal dans l'exemple (9) pour amener de façon emphatique un nouveau référent dans son foyer d'attention. L'emploi de la variante distale n'aurait pas eu cette valeur.

L'exemple (Iо), qui fait intervenir les deux membres de la paire this vs. that l'un après l'autre (mais en tant que pronoms cette fois), fait ressortir la distinction cognitivo-discursivo-interactionnelle qui les sépare de façon frappante:

(го) '( . . ) Fears have been raised that in their enthusiasm scientists might create a black hole or even something called 'strange quarks', which could, theoretically, interact with other subatomic particles and propagate uncontrollably.

${ }^{18}$ Voir entre autres à ce sujet, Langacker (2002a), qui la caractérise en termes de la 'perspective' ou du 'point de mire' adopté(e) sur le référent ciblé par le locuteur tout comme l'interlocuteur. 
If you are reading this, that hasn't happened...' (B. Bryson, 2004, A Short History of Nearly Everything, Doubleday, Black Swan, UK, p. 207)

L'emploi de la forme proximale this (ligne 3) peut s'analyser comme réalisant la procédure dite 'deixis textuelle', le pronom fonctionnant comme second argument du prédicat verbal 'read': this renvoie donc ici au segment de texte que le lecteur vient tout juste de lire au moment où il est utilisé (en l'occurrence, la première phrase de l'extrait). Il a de ce fait une valeur de deixis directe, mais le cadre ou 'fond' déictique ici est le texte précédent lui-même, et non la situation d'énonciation. En fait, c'est la résolution de son homologue distal that dans la principale qui facilite l'interprétation complète de this dans la protase, car il renvoie à la situation évoquée dans la première phrase de l'extrait: il ne s'agit donc pas du segment de texte qui contient immédiatement this.

En revanche, le pronom distal that, qui suit immédiatement this dans le fil du texte, a une fonction 'anadéictique "stricte ", car il renvoie à la situation hypothétique évoquée dans la première phrase. Remarquons la pertinence dans ce renvoi du contenu prédicatif de la proposition hôte ('hasn't happened'). La variante distale est employée ici, par hypothèse, car non seulement cette éventualité représente quelque chose qui est d'abord hypothétique, justement, et qui ensuite n'est pas souhaité (cf. la caractérisation en termes de 'craintes': ' . . . fears have been raised . . '); mais de plus, elle ne s'est même pas produite ('that hasn't happened').

Voici à présent un exemple où l'un ou l'autre des deux membres de l'opposition démonstrative pourrait être employé —mais avec un effet de sens très différent à chaque fois.

(i I) [Extrait d'un article 'St Tropez clean-up hits stars' hangout' à propos d'un restaurant de plage illégal sur la Côte d'Azur (The Guardian, 03/05/2000, p. 6)]

'(... ) But the council, fed up with complaints about the restaurant's loud music and the helicopters ferrying celebrities to and from a nearby helipad, is unlikely to let the matter rest.

"The courts will decide, but in theory all these beach bars and restaurants should be bulldozed", a spokesman said. "They are ugly, noisy, not terribly safe, and completely illegal. They have had a good run, but it is time to call it a day".'

Il s'agit ici d'une dispute entre le propriétaire d'un restaurant de plage (construit illégalement) et la municipalité qui cherche à le fermer. L'auteur présente en parallèle la position de chaque camp, mais ne prend pas position lui-même. Dans le cas de l'emploi par un porte-parole de la municipalité du SN démonstratif proximal quantifié all these beach bars and restaurants (ligne 4), notons d'abord qu'il aurait été possible d'employer à la place de these le déterminant distal those. Car la distinction 'proche/éloignée spatialement du locuteur' n'est pas opératoire dans le cas présent: en effet, le locuteur renvoie ici à une certaine conception des restaurants de plage (au niveau cognitif, donc), et non à tel ou tel restaurant au plan spatio-temporel.

En outre, dans le cadre de la conception plus interactive invoquée par Laury (I997, 2002) et Östman (I995) pour les types de marqueurs équivalents finnois, 
et par Cheshire (I996) et Glover (2000) à partir de données conversationnelles anglaises (voir aussi pour ses corrélats neurolinguistiques Kemmerer, I999), nous pouvons analyser cet emploi comme réalisant une valeur polémique en contexte, de la part du porte-parole du Conseil municipal. Dans cette optique, l'emploi de la forme proximale comporterait l'implication que sa conception du référent visé n'a pas encore été 'négociée' discursivement et ne fait donc pas partie du savoir partagé ('common ground') à l'œuvre. En effet, la perspective adoptée par le Conseil vis-à-vis de tels bars et restaurants est foncièrement négative, et à l'évidence son porte-parole ne peut pas s'attendre à ce que son opposant dans la dispute la partage. L'emploi du proximal these ici situe l'objet de discours à l'intérieur de la sphère subjective du locuteur, sphère supposée non partagée par ses interlocuteurs. Voir aussi Fillmore (I997) à ce sujet.

\section{APPROCHES EN TERMES DE 'SPHÈRES PERSONNELles' DeS INTERLOCUTEURS VS. 'DEgRÉS DE DistanCE SPATIALE DU RÉFÉRENT VISÉ PAR RAPPORT AU LOCUTEUR'}

À l'instar de la caractérisation des démonstratifs (déterminants comme pronoms) cist et cil de l'ancien français proposée par Marchello-Nizia (2005, 2006) en termes de 'renvoi à l'intérieur de la sphère subjective du locuteur' pour le proximal cist et de 'renvoi en dehors de la sphère subjective du locuteur' pour le distal cil, ${ }^{19}$ nombre de linguistes ont soutenu que les pronoms/déterminants this et that de l'anglais répondent également à une telle distinction, plutôt qu'à la distinction 'proximité/distance spatiale relative du référent visé par rapport au locuteur', comme le veut la tradition. Parmi ces linguistes se trouvent Cheshire (I996), Glover (2000), Strauss (2002) et Cornish (200I). Ces auteurs font valoir que la distinction traditionnelle entre deux valeurs de la dimension spatiale ou 'géographique' est en fait un cas spécial de la dichotomie discursivo-cognitive plus fondamentale entre la sphère subjective du locuteur, d'une part, et un détachement de cette sphère ainsi qu'un alignement avec celle de l'allocutaire, de l'autre.

L'approche 'spatiale' des marqueurs démonstratifs distingués pour la dichotomie proximale vs. distale achopperait sur l'exemple célèbre de Janssen (I995) que voici :

(I2) a. Docteur: Is this where it hurts? b. Patient: Yes, that is where it hurts. (néerlandais) Doet het zeer op deze plek? Ja, op die plek. (Janssen, I995: 249, ex. (2))

Comme le relève l'auteur, cette configuration des formes proximales et distales est le contraire de ce qui serait prédit par l'approche spatiale, car dans (I 2a), la partie du corps du patient pointée par le docteur ne pourrait pas être plus 'proche' du premier (objectivement parlant); pourtant, c'est son interlocuteur qui emploie la forme proximale this/deze; et inversement, le patient, lui, dans sa réponse en (I $2 \mathrm{~b}$ )

${ }^{19}$ Selon Guillot (2010: 237), la grande majorité des autres études de ces formes en ancien français tendent à privilégier l'explication 'spatiale' en termes du degré de proximité du référent visé par rapport au locuteur. 
emploie la forme distale that/die pour renvoyer indexicalement à cette même partie de son propre corps. Selon Janssen (p. 252), l'emploi de ces formes en (I 2a) serait motivé par le 'point de mire' ('subjective vantage point') impliqué à chaque fois. C'est la position de la main du docteur qui palpe le patient qui fait que cet endroit est au centre de son champ de vision et d'attention. Ce centre constitue de ce fait son 'focal concern', du point de vue de l'organisation de ce discours. L'emploi de la forme démonstrative distale par le patient pour renvoyer à ce même endroit de son corps en ( $\mathrm{I} 2 \mathrm{~b}$ ) refléterait interactivement la reconnaissance par le patient de la spécificité du champ de vision/d'attention de son interlocuteur.

Voir aussi l'argumentation et les indices que Kemmerer (I999) mobilise pour conclure que le fonctionnement des formes démonstratives proximales et distales n'est pas déterminé par des facteurs objectivement disponibles dans l'espace perceptif de la situation de communication, mais plutôt par 'a combination of the demonstrative's abstract semantic structure and the unique pragmatic conditions of the speech situation' (ibid., p. 52). Les données recueillies proviennent de plus de 80 langues (dont le français et l'anglais), tirées d'une gamme étendue de types de langues du monde. L'auteur mentionne de plus le fait que des analyses de la façon dont les démonstratifs proximaux et distaux sont employés dans les conversations indiquent que 'these terms have very broad functions which cannot be reduced to the restricted perceptual distinction between near and far regions of egocentric space' (ibid., p. 5I). Toutefois cet 'espace' n'est pas 'égocentrique', en réalité, déterminé à partir des coordonnées du seul locuteur, mais plutôt 'sociocentrique', dans la terminologie proposée par Jones (I995: 38): dans le cas de la deixis, comme dans ceux de l'anadeixis et de l'anaphore, il s'agit de l'établissement d'une coordination socio-cognitive complexe qui est à mettre en place parmi les interlocuteurs.

Du côté des études sociolinguistiques sur corpus, justement, Cheshire (I996: 372), en adoptant une variante de la dichotomie discursivo-cognitive en termes de 'sphères interlocutives', observe que, dans son corpus d'énoncés conversationnels anglais, 'the proximal-distal dimension [en termes du choix entre that vs. this et vice versa] is rarely relevant', 'the spatial dimension of their meaning [being] virtually always neutralised'. C'est également la conclusion à laquelle aboutit Glover (2000: 925), sur la base d'un corpus de négociations en urbanisme. Les analyses des exemples français et anglais proposées ci-dessus (cf. les $\S \S 2$ et 3 ) s'inscriraient également dans ce cadre.

A ce sujet, nous avons vu que les quatre exemples de ce $\mathrm{N}$-ci analysés en $\S 2$ (ce débat-ci en (2), cette fois-ci en (3), puis cette chambre-ci en (4a) et ce mois-ci en (4b)) témoignaient chacun d'une charge de subjectivité et d'implication personnelle de la part du scripteur et du locuteur vis-à-vis des objets respectifs de ces énonciations. De même, le souci de démarcation et de distanciation à l'égard des référents concernés était palpable chez les scripteurs qui employaient la forme distale ce $\mathrm{N}$-là dans (5) (ces moments-là), (6) (ce terroriste-là) et (7) (ce registre-là). Dans le cas de ces trois derniers marqueurs en ce $N$-là, l'alignement du scripteur ou du locuteur avec son lecteur ou allocutaire était également sensible. La même dichotomie s'appliquerait 
au fonctionnement des marqueurs proximaux et distaux anglais analysés en $\S_{3}$ : en effet, les occurrences de this ou these (N) en (8), (9), (Io) et (I I) étaient empreintes de subjectivité et d'emphase de la part des émetteurs, et celles de that (ou those) (N) dans ces mêmes exemples ${ }^{20}$ d'un souci d'alignement avec le destinataire ainsi que d'un désir de distanciation de la part des locuteurs ou des scripteurs.

Cette sensibilité des démonstratifs marqués pour la distinction proximale vs. distale cadrerait aussi avec l'approche cognitivo-discursive plus générale de l'anaphore, l'anadeixis et la deixis que j'essaie de développer depuis quelques années (cf. notamment Cornish, 20 Iоa, 20 I i voir aussi Kibrik, 20 I I et Pu, 20 I pour des conceptions comparables).

L'approche en termes de distinctions 'spatiales', en revanche, privilégie la perception directe de la situation et, par extension, les occurrences relatives des 'antécédents textuels' à l'œuvre dans le fil temporel du co-texte. Or, ce ne sont là que des inputs 'textuels' ${ }^{\prime 2}$ à la représentation mentale des entités concernées; le 'discours', lui (au sens plus 'mentaliste' de ce terme: voir plus bas), opère en termes de relations entre entités mentalement représentées, et non directement en fonction des éléments du 'texte', au sens large, selon la définition ci-dessous du concept. Comme le fait valoir Brisard (2002: xxiv) ' . . space and time cannot be treated as concepts in their own right but rather as modes or modalities of presentation.' Plus loin (p. xxix), l'auteur fait valoir que

[t]he focus on the subjective nature of grounding predications implies a conception of grammar that is not reflexive of contextual properties "out there" but of the speaker construing a context, possibly even creating it, and in any case designing it in function of the interaction with an addressee....

Voici en résumé ma conception de la triple distinction entre les dimensions du texte, du contexte et du discours et leurs interactions.

Selon moi, le texte (au sens 'massif, 'non-comptable' du terme, donc au sens " du texte ") est la trace d'au moins un acte d'énonciation —qu'il soit réalisé sous la forme d'une trace verbale, linguistique, ou bien non-verbale. En tant que tel, il comprend les traits paralinguistiques de ces actes, de même que des signaux nonverbaux sémiotiquement pertinents (cf. Clark, I996: ch. 6) tels que la direction du regard, le pointage gestuel et ainsi de suite. Le texte dans cette conception est essentiellement linéaire, à la différence du discours, qui est l'interprétation 'en ligne', continue et sujette à révision du texte, conjointement à un contexte approprié (qui doit inclure les intentions communicatives du locuteur, telles qu'elles sont inférées par l'allocutaire).

Le discours dépend à l'évidence à la fois du texte et du contexte. C'est le discours, et non (dans les conditions normales de son emploi en contexte) le texte qui est capable d'être stocké par la suite dans la mémoire à long terme, en attendant une récupération ultérieure possible. Par contre, la trace textuelle de l'événement

${ }^{20}$ Par substitution, uniquement, dans le cas de (9) et (I I).

${ }^{21} \mathrm{Au}$ sens large de ce terme que je préconise: voir Cornish (20Iоa) et plus bas. 
communicatif est éphémère (du moins à l'oral), car pour l'essentiel elle disparaît de la mémoire à court terme dès la construction du discours — ou peu de temps après.

S'agissant maintenant du contexte, j'en retiendrai sept aspects, dont le plus important est sans doute la situation d'énonciation, qui opère comme point d'ancrage par défaut pour le discours à construire: le domaine de référence d'un texte donné, le co-texte, le discours déjà construit en amont, le genre d'événement langagier en déroulement, le cadre socio-culturel supposé par le texte, les relations interlocutives existant entre les interactants à chaque moment de l'échange, et la situation d'énonciation particulière à l'œuvre. Le contexte est assujetti à un processus continu de construction et de révision au fur et à mesure que le discours se déroule. C'est à travers l'invocation d'un contexte approprié que l'auditeur ou le lecteur peut convertir en discours la séquence connexe d'indices perceptibles qu'est le texte. Or, ce contexte (qui est, comme le discours, mentalement représenté par les participants) ne pré-existe pas au discours de façon objective ou externe, mais est (re-)créé en permanence et en parallèle à chaque instant de la communication. Il est donc en développement constant: le discours construit à partir du texte en dépend, en même temps qu'il le modifie au fur et à mesure qu'il est créé en temps réel.

Or, la conception 'spatiale' de la distinction proximale/distale des démonstratifs ainsi marqués ne peut être envisagée de façon potentiellement plausible que lors de l'emploi de la procédure déictique canonique, en situation: car c'est là que l'on pourrait (en principe) caractériser les référents perçus par les interlocuteurs en fonction de leur degré de proximité relative du locuteur (ou de l'allocutaire, voire de l'ensemble des deux). Mais même là, nombre d'études de la communication langagière en situation relèvent des emplois des démonstratifs qui ne se conforment pas à cette hypothèse: voir à cet égard les renvois à Cheshire (I996) et à Glover (2000) plus haut, ainsi que Hanks (2009). De même, lorsque les référents des marqueurs concernés sont abstraits et non concrets, aucune distinction 'spatiale' en termes de proximité ou d'éloignement du locuteur ne peut être invoquée —et cependant, les formes démonstratives proximales et distales les désignent tout comme les objets concrets (cf. aussi Debruyn, I992: 28). De plus, hors de l'emploi en situation, et notamment à l'écrit, l'espace perceptif que constitue la situation d'énonciation ne sera pas disponible en tant que tel.

Toutefois, les partisans de la conception spatiale diront que dans un texte écrit (ou dans un récit à l'oral, par exemple), le paramètre spatial est réinterprété en tant que paramètre temporel; ainsi, les formes démonstratives proximales cibleraient les objets de discours mentionnés plus récemment dans le co-texte (étant relativement plus 'proches', temporellement, du locuteur, donc), alors que les formes distales s'orienteraient vers ceux exprimés plus avant dans le déroulement du texte. Mais encore une fois, cette conception ne cadre pas toujours avec la manière réelle dont les interlocuteurs emploient les démonstratifs en contexte. Il est vrai que les démonstratifs proximaux français en ce $N$-ci illustrés en (2) et (3) pourraient être analysés de cette façon: cependant, nous avons vu qu'il y avait d'autres facteurs, 
plus fondamentaux, à l'œuvre dans chacun de ces cas — facteurs qui primeraient sur ces notions d'ordre d'apparition relative dans le co-texte.

S'agissant des emplois de la forme démonstrative distale ce $N$-là des exemples (5)(7), dans (5), ce marqueur fonctionne en terme de la situation évoquée par l'image, pour cibler par inférence un type d'occasion (les obsèques); il s'agit alors d'une instanciation de la procédure anadéictique dite 'de reconnaissance' ('mémorielle'). En tant que telle, ni la conception en termes du paramètre spatial, ni celle en fonction du paramètre temporel, ne serait de mise. Et dans le cas de la forme distale dans l'exemple (6), la situation représente le contraire de ce qui serait prédit par l'hypothèse spatiale réinterprétée en termes d'ordre de mention temporelle. Car le SN démonstratif distal ce terroriste-là renvoie au type nouveau de terroriste qui vient d'être caractérisé, et non à celui qui pourrait correspondre concrètement aux deux frères mentionnés plus tôt dans cet extrait. Dans (7) également, bien qu'il n'y ait pas 'compétition' entre deux entités de même type dans le co-texte précédent, la forme distale ce registre-là renvoie à un type de description de l'individu concerné qui vient tout juste d'être caractérisée. Dans ces trois cas, l'emploi de la forme démonstrative proximale n'aurait pas été possible, ou du moins naturel, avec la même interprétation et référence que son homologue distal.

De même, dans le cas des cinq occurrences de formes démonstratives anglaises que nous avons vues dans (8)-(I I), la conception spatiale - même réinterprétée en termes temporels — n'aurait pas été d'une grande utilité pour prédire et expliquer le choix du membre des paires de marqueurs sélectionné, et l'impossibilité — ou du moins la différence de référence et d'interprétation à laquelle cette alternative donnerait lieu — dans ces contextes du membre opposé.

\section{POUR CONCLURE...}

En conclusion, selon nous la particule française $-c i$ retient de son ancêtre ici la préférence d'un fonctionnement déictique en contexte; cependant, en se grammaticalisant, il y aurait une légère diminution de la valeur déictique pure associée à l'emploi de l'adverbe démonstratif proximal. En conséquence, cette particule aurait dans la langue contemporaine de préférence un fonctionnement anadéictique, où la dimension déictique primerait toutefois sur l'anaphorique. C'est cela qui fait que, à la fois dans l'analyse de De Mulder et al. (20I0) du français médiéval, dans celle de Debruyn (I992) et de bien d'autres linguistes de la langue actuelle, son interprétation est contrainte à s'inscrire par priorité dans les circonstances immédiates de son occurrence - circonstances prises en charge donc totalement par l'énonciateur. Par contre, la particule distale -là, quand l'opposition entre -ci et -là n'est pas neutralisée, serait vouée à trouver son interprétation par le truchement d'éléments extérieurs à ces circonstances immédiates. Elle a donc vocation à cibler des informations ou des entités mutuellement connues, déjà négociées et partagées par les interlocuteurs (ou présumées telles). Les notions de 'proximité' et d' 'éloignement' spatio-temporels vis-à-vis du locuteur ou de 
l'allocutaire privilégiées par la tradition seraient de ce fait prédites et anticipées par cette distinction plus cognitivo-discursivo-interactionnelle.

Le fonctionnement de la 'descendante' contemporaine de l'opposition cist/cil en ancien français créée par la langue pour la suppléer dès le Moyen Âge, à savoir celle entre $c e . . .-c i$ et $c e . .$. -là, n'est donc pas tributaire de distinctions purement spatiales ou temporelles du référent en relation avec le locuteur; il conserve plutôt une variante de la distinction 'à l'intérieur de la sphère subjective du locuteur' / en dehors de la sphère subjective du locuteur' de son ancêtre. Nous pourrons postuler que ce sont les particules suffixées -ci et -là, issues elles-mêmes des adverbes lexicaux ici et là, respectivement, qui ont en quelque sorte 'pragmaticalisé', en se grammaticalisant, cette distinction ancienne. Pour Guillot (20 I0: 245), jusqu'en français médiéval, les emplois de cist et de cil répondraient à la présence vs. l'absence, respectivement, d'une prise en charge personnelle, subjective du locuteur vis-à-vis de son référent, tout en prenant position par rapport à son allocutaire ou lecteur. Dans les mots de l'auteur:

Nous avons proposé de reconsidérer la valeur de CIST et CIL en ancien français relativement au trait " $+/$ - prise en charge du contenu informatif du SN par le locuteur ", la série CIST étant utilisée lorsque le locuteur assume la responsabilité de l'énonciation du SN, la série CIL dans la situation inverse.

C'est bien entendu l'opposition 'marqué' (les formes proximales) vs. 'nonmarqué' (les formes distales) qui fonde cette distinction. La citation qu'on vient de voir le montre bien, car alors que cist (membre proximal) serait marqué positivement pour le trait " prise en charge du contenu informatif du SN par le locuteur ", cil (membre distal) ne le serait pas. Nous pouvons postuler en fait qu'en tant que membre non marqué de l'opposition concernée, cil la neutralise. Partant, par hypothèse, les membres distaux des paires de marqueurs indexicaux que nous avons traités ci-avant neutraliseraient également les oppositions concernées, en tant que membres non marqués de chaque paire, précisément. ${ }^{22}$

Une fois opéré le choix de l'une ou l'autre de ces formes, en fonction de cette distinction systémique, le contexte à l'œuvre déterminera la valeur précise que revêtira celle qui aura cours. L'opposition anglaise des démonstratifs this $(N)$ vs. that $(N)$, qui sont également marqués pour la distinction proximal vs. distal, fonctionnerait selon une dichotomie voisine: car l'emploi des formes démonstratives proximales plus généralement (here, now, this $(N)$ ) exprimerait l'implication personnelle, subjective de la part du locuteur avec leur référent; alors que celui des formes distales (there, then, that $(N)$ ) supposerait soit la dissociation personnelle, subjective du locuteur par rapport au référent, soit un alignement entre locuteur et allocutaire à cet égard, où l'entité visée serait conçue comme relevant d'une information déjà négociée, en termes interactionnels. C'est précisément en termes interactionnels, en fonction de la dynamique du discours en déroulement

${ }^{22}$ Cf. la discussion au tout début de la section 2 , ainsi que les notes 4 et 5 , la description de l'évolution des démonstratifs français chez Guillot (à paraître, 2015), puis la caractérisation par Lyons (1975) des formes démonstratives distales that et there de l'anglais. 
et de la coordination des perceptions et représentations mentales des interlocuteurs en présence, que cette opposition devrait être envisagée.

L'emploi des formes démonstratives marquées pour la distinction proximal vs. distal dans ces deux langues (mais dans d'autres également: voir par exemple les allusions aux faits du néerlandais et du finnois dans la section 4) n'est donc pas tributaire de facteurs extra-discursifs — au sens de ce terme employé ici —, purement 'métriques'. Il est plutôt lié au projet communicatif des interlocuteurs - ou du scripteur vis-à-vis de son lecteur dans le cas de la communication écrite. C'est le sujet parlant ou écrivant qui commande, en liaison avec son allocutaire ou son lecteur, non des facteurs qui lui sont externes.

Adresse pour correspondance:

Laboratoire CLLE-ERSS

Maison de la Recherche

Université de Toulouse Jean Jaurès

5, Rue Antonio Machado

31058 Toulouse Cedex 9

France

E-mail:cornish@univ-tlse2.fr

\section{RÉFÉRENCES}

Ariel, M. (1998). The linguistic status of the "here and now". Cognitive Linguistics, 9.3: I $89-237$.

Béguelin, M.-J. (1998). L'usage des SN démonstratifs dans les Fables de La Fontaine. Langue Française, I20: 95-109.

Brisard, F. (2002). Introduction: The epistemic basis of deixis and reference. In: Brisard, F. (dir.), Grounding. The Epistemic Footing of Deixis and Reference. Berlin et New-York: Mouton de Gruyter, pp. xii-xxxiv.

Bühler, K. (2009)/[I934]. Théorie du langage. La fonction représentationnelle du langage. Traduction, notes et glossaire par Didier Samain. Collection Banc d'Essais. Marseille: Agone.

Cheshire, J. (1996). That jacksprat: an interactional perspective on English that. Journal of Pragmatics, 25: 369-393.

Clark, H. H. (I996). Using Language. Cambridge: Cambridge University Press.

Cornish, F. (200I). "Modal" that as determiner and pronoun: the primacy of the cognitive-interactional parameter. English Language and Linguistics, 5.2: 297-3 I 5 .

Cornish, F. (2010a). Anaphora: Text-based or discourse-dependent? Functionalist vs. formalist accounts. Functions of Language, I7.2: 207-24I.

Cornish, F. (20Iob). Indexicaux, discours, et mémoire discursive. Ce que les premiers révèlent du second et de la troisième. LINX, 62-63: I I I-I33.

Cornish, F. (20II). 'Strict' anadeixis, discourse deixis and text structuring. Language Sciences, 33.5: 753-767.

Debruyn, J. (I992). L'opposition de $\mathrm{Ce}+\mathrm{N}-\mathrm{ci} / \mathrm{Ce}+\mathrm{N}$-là: un double fonctionnement. UIA, Anvers (Antwerp Papers in Linguistics 68). 


\section{Francis Cornish}

De Mulder, W. et Carlier, A. (2006). Du démonstratif à l'article défini: le cas de ce en français moderne. Langue Française, I 52: 96-1 I3.

De Mulder, W., Guillot, C. et Mortelmans, J. (2010). Ce N-ci et ce N-là en moyen français. In: Tovena Lucia, M. (dir.), Déterminants en diachronie et en synchronie. Paris: Projet ELICO Publications, pp. 86-IO3.

De Mulder, W. et Vetters, C. (2008). Le sens fondamental de maintenant: un tokenreflexive. Cahiers Chronos, 20 : I 5-33.

Diessel, H. (1999). Demonstratives. Form, Function, and Grammaticalization. Amsterdam/Philadelphia: John Benjamins.

Ehlich, K. (1982). Anaphora and deixis: Same, similar, or different? In: R. J. Jarvella et W. Klein (dir.), Speech, Place and Action. Studies in Deixis and Related Topics. Chichester: Wiley, pp. 3I 5-338.

Fillmore, C. J. (1997). Lectures on Deixis. Stanford, CA: CSLI.

Forget, D. (1989). Là : un marqueur de pertinence discursive. Revue Québécoise de Linguistique, I8.1: 57-82.

Glover, K. (2000). Proximal and distal deixis in negotiation talk. Journal of Pragmatics, 32: 9I 5-926.

Guillot, C. (2010). Les démonstratifs de l'ancien français: un système encore personnel? In: F. Neveu, V. Muni Toke, J. Durand, T. Klingler, L. Mondada et S. Prévost (dir.) Congrès Mondial de Linguistique Française - CMLF 2010 9782-7598-0534-I, Paris, 20I0, Institut de Linguistique Française, EDP Sciences (www.linguistiquefrancaise.org), [http://dx.doi.org/I0.I05 I/cmlf/20I0085].

Guillot, C. (20I3). Système des démonstratifs médiévaux et exemples de stratégies communicatives. Journal of French Language Studies, 23.2: 22 I-242.

Guillot, C. (à paraitre, 2015). From Old French and Middle French to Contemporary French. In: K. Jungbluth et F. Da Milano (dir.), Manual of Deixis in Romance Languages. Berlin/Boston: Mouton de Gruyter, pp. 546-568.

Hanks, W.F. (2009). Fieldwork on deixis. Journal of Pragmatics, 4I: IO-24.

Himmelmann, N.P. (I996). Demonstratives in narrative discourse: a taxonomy of universal uses. In: B. A. Fox (dir.), Studies in Anaphora. Amsterdam/Philadelphia: John Benjamins, pp. 205-254.

Janssen, T.A.J.M. (1995). Deixis from a cognitive point of view. In: E. Contini-Morava et B. S. Goldberg (dir.), Meaning as Explanation: Advances in Linguistic Sign Theory. Berlin: Mouton de Gruyter, pp. 245-270.

Jones, P. (1995). Philosophical and theoretical issues in the study of deixis: A critique of the standard account. In: K. Green (dir.), New Essays in Deixis. Discourse, Narrative, Literature. Amsterdam/Atlanta: Rodopi, pp. 27-48.

Kemmerer, D. (I999). "Near" and "far" in language and perception. Cognition, 73: $35-63$.

Kibrik, A. A. (20I I). Reference in Discourse. New York/Oxford: Oxford University Press.

Kleiber, G. (1990). Sur l'anaphore démonstrative. In: M. Charolles, S. Fisher et J. Jayez (dir.), Le discours. Représentations et interprétations, Nancy: Presses Universitaires de Nancy, pp. 243-263.

Kleiber, G. (I994). Anaphores et pronoms. Louvain-la-Neuve: Duculot.

Kleiber, G. (2008). Comment fonctionne ICI ? Cahiers Chronos, 20: I I $3-$ I 45 .

Langacker, R.W. (2002a). Deixis and subjectivity. In: F. Brisard (dir.), Grounding. The Epistemic Footing of Deixis and Reference. Berlin et New York: Mouton de Gruyter, pp. I-27. 
Langacker, R. W. (2002b). Remarks on the English grounding systems. In: F. Brisard (dir.), Grounding. The Epistemic Footing of Deixis and Reference. Berlin et New York: Mouton de Gruyter, pp. 29-38.

Laury, R. (1997). Demonstratives in Interaction. The Emergence of a Definite Article in Finnish. Amsterdam/Philadelphia: Benjamins.

Laury, R. (2002). Interaction, grounding and third-person referential forms. In: F. Brisard (dir.), Grounding. The Epistemic Footing of Deixis and Reference. Berlin et New York: Mouton de Gruyter, pp. 83-i I I.

Le Draoulec, A. (2013). Des adverbes entre espace et temps. Le cas singulier de ici, d'ici, jusqu'ici. Faits de Langue, 42: 87-I07.

Lyons, J. (1975). Deixis as the source of reference. In: E. L. Keenan (dir.), Formal Semantics of Natural Language. Cambridge: Cambridge University Press, pp. 6I-83.

Marchello-Nizia, C. (2005). Deixis and subjectivity: the semantics of demonstratives in Old French (9th-12th century). Journal of Pragmatics, 37: 43-68.

Marchello-Nizia, C. (2006). Du subjectif au spatial: l'évolution des formes et du sens des démonstratifs en français. Langue Française, I 52: I 14-126.

Östman, J-O. (1995). Recasting the deictic foundation, using Physics and Finnish. In: M. Shibatani et S. A. Thompson (dir.), Essays in Semantics and Pragmatics in Honor of Charles J. Fillmore. Amsterdam/Philadelphia: Benjamins, pp. 247-278.

Prince, E. F. (I98I). On the inferencing of indefinite-this NPs. In: A. K. Joshi, B-L. Webber et I. A. Sag (dir.), Elements of Discourse Understanding. Cambridge: Cambridge University Press, pp. $23 \mathrm{I}-250$.

Pu, M.-M. (20I I). Discourse Anaphora: A Cognitive-functional Account. Munich: Lincom Europa.

Schiffrin, D. (1990). Between text and context: Deixis, anaphora and the meaning of then. Text, I0.3: $245-270$.

Strauss, S. (2002). This, that and it in spoken American English: a demonstrative system of gradient focus. Journal of Pragmatics, 24: I 3 I-I 52.

Theissen, A. (2008). Le SN démonstratif cette fois (-ci/-là). Journal of French Language Studies, 18: 209-226. 